\title{
VOID SHAPES AND o-Ps LIFETIME IN MOLECULAR CRYSTALS
}

\author{
B. JASIŃSKA ${ }^{a}$, A.E. KOZIOE ${ }^{b}$ AND T. GowOREK ${ }^{a}$ \\ ${ }^{a}$ Institute of Physics, ${ }^{b}$ Department of Crystallography, Institute of Chemistry \\ Maria-Curie Skłodowska University, 20-031 Lublin, Poland
}

The size and shape of the free volume in molecular crystals was estimated from crystallographic data. The o-Ps lifetime values were calculated from respective models and compared with the experimental data. The best agreement for channel-like voids has been obtained for the value of $D R$ parameter equal $0.19 \mathrm{~nm}$. The threshold value of radius allowing $P$ s trapping in the void was found to be $(0.12-0.13) \mathrm{nm}$.

PACS numbers: $78.70 . \mathrm{Bj}$

\section{Introduction}

A well-known model by Tao [1] and Eldrup et al. [2] allows us to estimate the $o$-Ps lifetime in the spherical potential well assuming an infinite rectangular barrier; the penetration of the Ps wave function into the bulk is simulated by extension of the well size by $D R$. The pick-off lifetime is then

$$
\tau^{\mathrm{sph}}=\left[\lambda_{a}\left(1-\frac{R}{R_{0}}+\frac{1}{2 \pi} \sin 2 \pi \frac{R}{R_{0}}\right)\right]^{-1},
$$

where $R$ - free volume radius, $R_{0}=R+D R$. The value of empirical parameter $D R$ is usually assumed $0.166 \mathrm{~nm}[2]$, and $\lambda_{a}=2 \mathrm{~ns}$ is the spin averaged decay rate.

Sometimes the free volumes, particularly in crystalline solids, have an elongated form, e.g., vacancies in long-chain compounds or natural channel-like free volumes running through the crystal structure. Such voids can be approximated by cylinders or cuboids. For the case of cuboid the appropriate formula is:

$$
\dot{\tau}^{\mathrm{cub}}=\left\{\lambda_{a}\left[1-\prod_{i=1}^{3}\left(\frac{a_{i}}{a_{i}+2 \Delta R}+\frac{1}{\pi} \sin \frac{\pi a_{i}}{a_{i}+2 \Delta R}\right)\right]\right\}^{-1},
$$

where $a_{1}, a_{2}, a_{3}$ - cuboid sides. The same Eq. (2) can be used for channels with rectangular cross section $\left(a_{3} \rightarrow \infty\right)$ and for layered structures $\left(a_{2}=a_{3} \rightarrow \infty\right)$. For infinite cylindrical channels

$$
\tau^{\mathrm{cyl}}=\left(1.28 \lambda_{a} \int_{X R / R_{0}}^{X} J_{0}^{2}(r) r \mathrm{~d} r\right)^{-1}
$$

( $J_{0}$ is the cylindrical Bessel function, $X$ - the lowest node of this function). 


\section{Results and discussion}

We have determined the free volume sizes from the crystallographic data for a set of molecular crystals and then compared the expected o-Ps lifetime with experimental results. The atom sizes were assumed to be equal to the radii at which the electron density at the outermost orbital reaches its maximum. The free volume was determined by inscribing the bodies (e.g. spheres) tangent to the surrounding atoms. In several cases we have found that positronium is not formed at all, in other cases the observed o-Ps lifetime lies in the range of (0.6-1.2) ns.

\subsection{Spherical voids}

There are very close packed crystals as fluorathene and acenaphtylene in which, at our atoms size definition, one can inscribe a sphere with a radius of $\cong 0.05 \mathrm{~nm}$ only. In these crystals we did not observe positronium formation. In the structure of azulene and anthracene the free volumes are bigger $(0.10 \mathrm{~nm}$ and $0.115 \mathrm{~nm}$, respectively) but Ps is still not formed (Fig. 1). The threshold of Ps formation is located somewhere near a free volume radius of $(0.12-0.13) \mathrm{nm}$, but its exact location depends also on the values of positron and electron energies for each of the solids studied. Thus, at this value of threshold radius we should not expect in molecular crystals the $o$-Ps lifetimes shorter than $\sim 750$ ps.

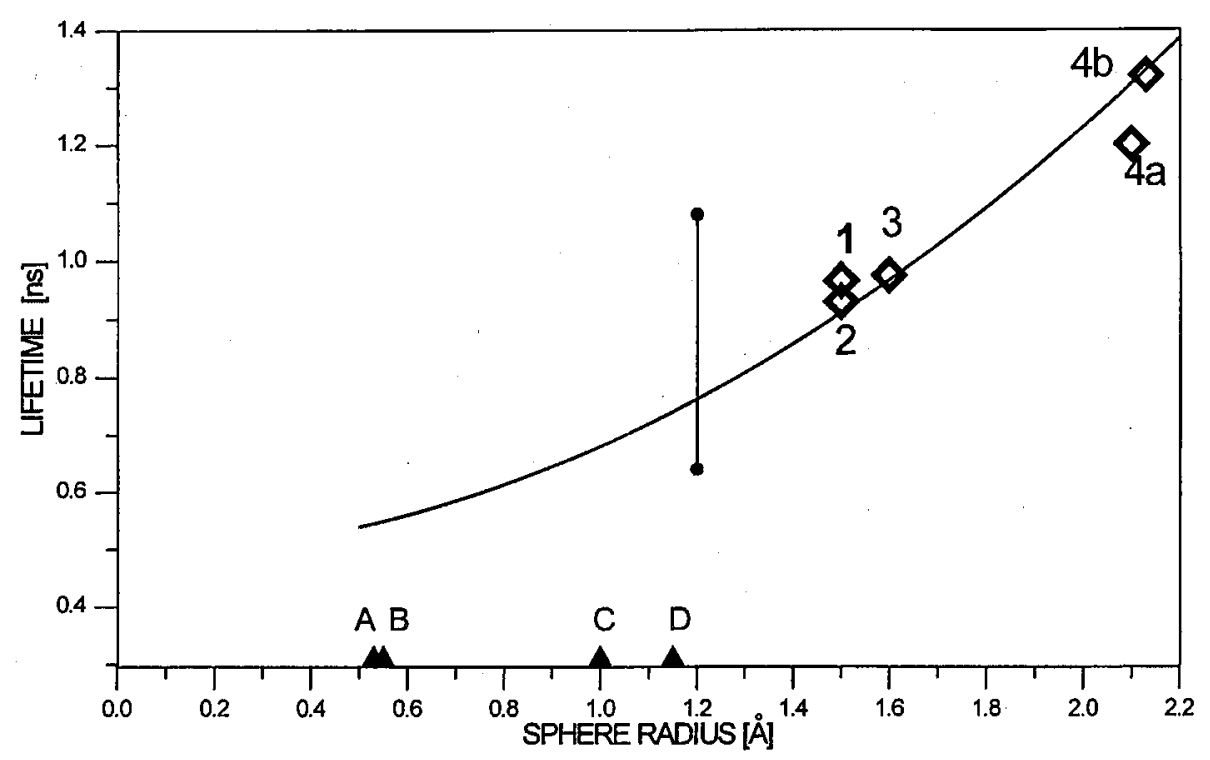

Fig. 1. The o-Ps lifetimes in some molecular crystals with spherical free volumes, compared with Tao-Eldrup model. The vertical bar denotes the limits of the lifetime in $p$-terphenyl when the temperature changes from 400 to $460 \mathrm{~K}$. Letters and numbers denote: A - fluoranthene, B - acenaphtylene, C - azulene, D - anthracene, 1 - naphthalene at $239 \mathrm{~K}, 2$ - resorcinol, 3 - chrysene, $4 \mathrm{a}$ - benzene at $138 \mathrm{~K}$, $4 \mathrm{~b}-$ benzene at $218 \mathrm{~K}$. Lifetime values taken from Refs. [6-9]. 


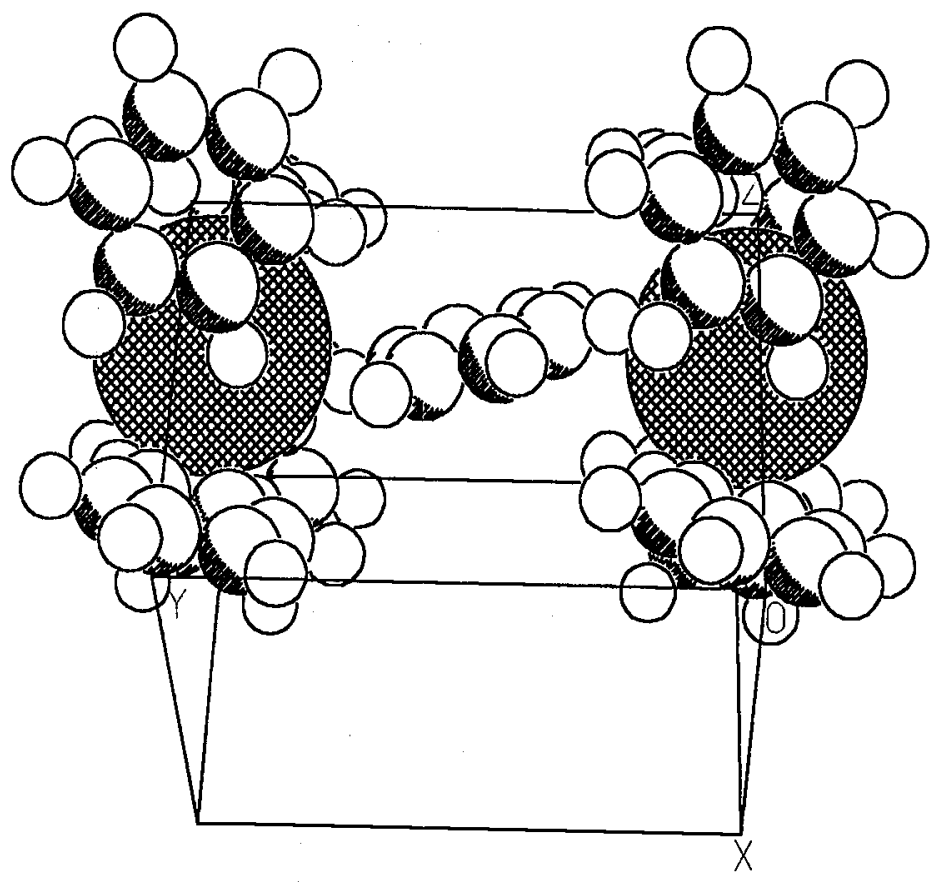

Fig. 2. Crystal cell of solid benzene with the sphere approximating the free volume. Atom positions taken from Ref. [4].

In Fig. 1 one can see that the o-Ps lifetimes estimated from the model agree well with the experimental values of $R$ up to $0.21 \mathrm{~nm}$ (or $1.3 \mathrm{~ns}$ ). Figure 2 shows the sphere inscribed into the structure of benzene crystal; the location of free volume in some other crystals was shown in our previous paper [3].

All crystallographic and lifetime data in Fig. 1 (and in this whole paper) relate to the room temperature, except of naphtalene $(239 \mathrm{~K})$ and benzene $(138 \mathrm{~K}$ and $218 \mathrm{~K}$ ).

\subsection{Cuboidal voids}

In some crystals the free space has cuboidal or even channel-like structure. In that case, inscribing the sphere in the manner as described above we always obtain the lifetime value too low in comparison to the experimental one (Table).

The model calculations of 0 -Ps lifetime in cuboids are shown in Fig. 3. One can see that, if the cross section of cuboid is constant and third axis is lengthening, the $t$ value is also increasing, but tends rapidly to the saturation value, which corresponds to the channel-like structure. It is interesting that a sphere $(D R=$ $0.166 \mathrm{~nm}$ ) having the same diameter as the cube side (with $D R=0.190 \mathrm{~nm}$ ) gives almost the same lifetime value. It is seen from Table that all values calculated assuming $D R=0.166 \mathrm{~nm}$ are a bit higher than the experimental ones. Recently we have proposed for cylindrical capillaries in porous materials $D R$ value equal $0.19 \mathrm{~nm}$ [5]. If we apply it to estimate the lifetime from Eq. (2) we can observe excellent agreement between experiment and model. 


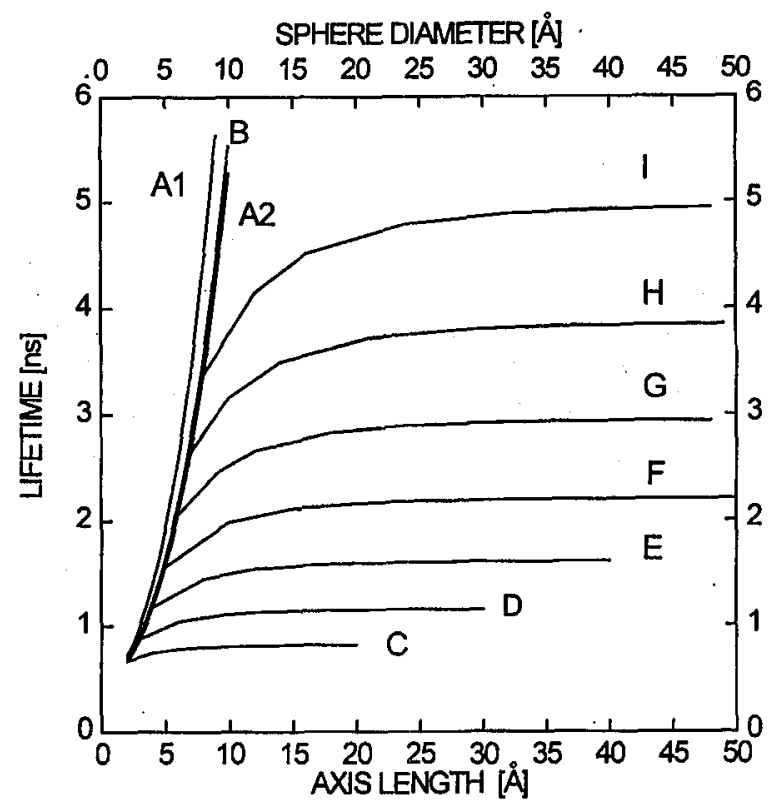

Fig. 3. The o-Ps lifetimes in cuboidal voids compared to sphere and cube. Respective curves denote: $\mathrm{A} 1$ - cube with $D R=0.166 \mathrm{~nm}, \mathrm{~A} 2-$ cube with $D R=0.19 \mathrm{~nm}$, $\mathrm{B}-$ sphere, $\mathrm{C}$ to $\mathrm{I}$ cuboid of constant square cross-section $(\mathrm{C}-0.2 \times 0.2 \mathrm{~nm}, \mathrm{D}-$ $0.3 \times 0.3 \mathrm{~nm}, \mathrm{E}-0.4 \times 0.4 \mathrm{~nm}, \mathrm{~F}-0.5 \times 0.5 \mathrm{~nm}, \mathrm{G}-0.6 \times 0.6 \mathrm{~nm}, \mathrm{H}-0.7 \times 0.7 \mathrm{~nm}$, $\mathrm{I}-0.8 \times 0.8 \mathrm{~nm})$.

\section{TABLE}

Ortho-Positronium lifetimes in the molecular crystals with channel-like free volumes.

\begin{tabular}{l|c|c|c|c}
\hline \hline \multirow{2}{*}{ Crystal } & \multicolumn{4}{|c}{ Lifetime [ns] } \\
\cline { 2 - 5 } & \multirow{3}{*}{ Experimental } & Sphere & Cuboid & Cuboid \\
\cline { 3 - 5 } & & $D R=$ & $D R=$ & $D R=$ \\
$0.166 \mathrm{~nm}$ & $0.166 \mathrm{~nm}$ & $0.190 \mathrm{~nm}$ \\
\hline catechol & $0.98 \pm 0.02[9]$ & 0.72 & 1.24 & 1.08 \\
phenanthrene & $1.08 \pm 0.02[10]$ & 0.68 & 1.18 & 1.05 \\
hydroquinone & $1.08 \pm 0.01[9]$ & 0.91 & 1.26 & 1.10 \\
biphenyl & $1.14 \pm 0.01[8]$ & 0.84 & 1.32 & 1.14
\end{tabular}

\section{Conclusions}

In the case of free volumes which can be approximated by spheres there is relatively good agreement between experimental o-Ps lifetime and the estimations based on the crystallographic data. The observed o-Ps lifetimes in channel-like free volumes are systematically shorter than those estimated from Eq. (2); one can obtain good agreement using the modified value $D R=0.19 \mathrm{~nm}$. 


\section{References}

[1] S.J. Tao, J. Chem. Phys. 56, 5499 (1972).

[2] M. Eldrup, D. Lightbody, J.N. Sherwood, Chem. Phys. 63, 51 (1981).

[3] B. Jasińska, A.E. Kozioł, T. Goworek, J. Radioanal. Nucl. Chem. 210, 617 (1996).

[4] G.E. Bacon, N.A. Curry, S.A. Wilson, Proc. R. Soc. Lond. Ser. A 279, 98 (1964).

[5] K. Ciesielski, A.L. Dawidowicz, T. Goworek, B. Jasińska, J. Wawryszczuk, Chem. Phys. Lett. 289, 41 (1998).

[6] T. Goworek, C. Rybka, R. Wasiewicz, J. Wawryszczuk, Phys. Status Solidi B 113, K9 (1982).

[7] P.C. Jain, M. Eldrup, J.N. Sherwood, in: Positron Annihilation, Eds. P.G. Coleman, S.C. Sharma, L.M. Diana, North Holland, Amsterdam 1982, p. 674.

[8] T. Goworek, C. Rybka, J. Wawryszczuk, R. Wasiewicz, Chem. Phys. Lett. 106, 482 (1984).

[9] T. Goworek, B. Jasińska, J. Wawryszczuk, K. Ciesielski, J. Chem. Soc. Faraday Trans. 93, 1573 (1997).

[10] W. Górniak, T. Goworek, C. Rybka, Chem. Phys. Lett. 187, 537 (1991). 